\title{
Monogenic gene variants in lung transplant recipients with usual interstitial pneumonia
}

\author{
Christoffer Stark $\mathbb{B}^{1}$, Juha W. Koskenvuo ${ }^{2}$, Antti Nykänen ${ }^{1}$, Eija H. Seppälä ${ }^{2}$, Samuel Myllykangas ${ }^{2}$, \\ Karl Lemström (i) ${ }^{1}$ and Peter Raivio ${ }^{1}$
}

${ }^{1}$ Heart and Lung Center, Helsinki University Hospital and University of Helsinki, Helsinki, Finland. ${ }^{2}$ Blueprint Genetics Ltd, Helsinki, Finland.

Corresponding author: Christoffer Stark (christoffer.stark@hus.fi)

\begin{abstract}
Shareable abstract (@ERSpublications)
A molecular diagnosis is common in lung transplant recipients with usual interstitial pneumonia and frequently reveals variants in genes related to telomerase function. This finding is not associated with increased risk of allograft dysfunction. https://bit.ly/30ucMQy
\end{abstract}

Cite this article as: Stark C, Koskenvuo JW, Nykänen A, et al. Monogenic gene variants in lung transplant recipients with usual interstitial pneumonia. ERJ Open Res 2022; 8: 00583-2021 [DOI: 10.1183/23120541.00583-2021].

Copyright (The authors 2022

This version is distributed under the terms of the Creative Commons Attribution NonCommercial Licence 4.0. For commercial reproduction rights and permissions contact permissions@ersnet.org

Received: 13 Oct 2021

Accepted: 9 Dec 2021

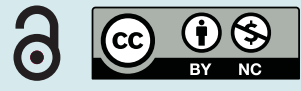

\section{Abstract}

Aim The prevalence of monogenic disease-causing gene variants in lung transplant recipients with idiopathic pulmonary fibrosis is not fully known. Their impact on clinical outcomes before and after transplantation requires more evidence.

Patients and methods We retrospectively performed sequence analysis of genes associated with pulmonary fibrosis in a cohort of 23 patients with histologically confirmed usual interstitial pneumonia that had previously undergone double lung transplantation. We evaluated the impact of confirmed molecular diagnoses on disease progression, clinical outcomes and incidence of acute rejection or chronic lung allograft dysfunction after transplantation.

Results 15 patients out of 23 (65\%) had a variant in a gene associated with interstitial lung disease. 11 patients (48\%) received a molecular diagnosis, of which nine involved genes for telomerase function. Five diagnostic variants were found in the gene for Telomerase reverse transcriptase. Two of these variants, p.(Asp684Gly) and p.(Arg774*), seemed to be enriched in Finnish lung transplant recipients. Disease progression and the incidence of acute rejection and chronic lung allograft dysfunction was similar between patients with telomere-related disease and the rest of the study population. The incidence of renal or bone marrow insufficiency or skin malignancies did not differ between the groups.

Conclusion Genetic variants are common in lung transplant recipients with pulmonary fibrosis and are most often related to telomerase function. A molecular diagnosis for telomeropathy does not seem to impact disease progression or the risk of complications or allograft dysfunction after transplantation.

\section{Introduction}

Idiopathic pulmonary fibrosis (IPF) is a chronic and progressive interstitial lung disease with unknown aetiology in most cases [1]. A diagnosis for usual interstitial pneumonia (UIP), the most common type of IPF, is based on typical findings on high-resolution computed tomography and from surgical biopsies [2]. Median survival of patients with IPF is generally $<5$ years [3]. There is considerable variation in disease progression with sudden exacerbation occurring in some patients [4]. Antifibrotic therapy might improve survival and slow down progression but so far, there are no curative treatment strategies for IPF [5]. Lung transplantation is often the only viable treatment option for these patients. Globally, 22-50\% of lung transplantations are performed for interstitial lung disease [1, 6, 7]. After lung transplantation, rejection and chronic lung allograft dysfunction (CLAD) are the main reasons for graft loss and mortality [8].

The cellular mechanisms responsible for disease progression in IPF are sustained epithelial injury, accelerated epithelial ageing, and promotion of interstitial fibrosis by interplay between inflammatory cells and myofibroblasts. Factors such as cigarette smoke, air pollution, microaspiration and infectious agents 
are possible triggers for these effects [1, 9]. Genetic factors contribute to the pathogenesis of IPF either via Mendelian inheritance or by increasing disease susceptibility via complex mechanisms. Monogenic disease forms are most commonly associated with genes regulating telomerase function [10]. Telomerase is responsible for maintaining telomere length. Shortened telomeres can lead to several clinical phenotypes, and the most common manifestation of adult-onset telomere disease is pulmonary fibrosis [11].

Utilisation of genetic testing is increasing even though it is not routinely used in the clinical workup of patients with suspected or confirmed IPF nor in patients listed for lung transplantation. Identification of a molecular diagnosis for IPF might allow earlier diagnosis and therapeutic interventions, as well as offer possibilities for genetic counselling and familial risk assessment. Surgical biopsies could also be avoided in patients with a clear molecular IPF diagnosis [12]. A molecular diagnosis of telomeropathy has been associated with bone marrow failure and liver dysfunction post-transplant, but there is conflicting evidence whether pathological genetic variants affect allograft dysfunction [13, 14]. Our objective was to clarify monogenic disease burden in lung transplant recipients and to identify whether genetics influence disease progression or the incidence of acute rejection and CLAD after transplantation.

Material and methods

Study subjects, study design and patient management

For this study, we included IPF patients who underwent de novo lung transplantation at Helsinki University Hospital between January 2007 and December 2015. Patients had to be alive at the time of recruitment (January 2019) in order to donate DNA samples. We identified 59 transplant recipients meeting these criteria of which 38 patients had histologically confirmed UIP. These patients were invited to participate in the study. 24 patients gave their written informed consent and donated saliva for DNA extraction and analysis. One sample was contaminated, and DNA extraction was not possible. For final analysis, 23 patients were included and followed until December 2019. The ethical review board at Helsinki University Hospital approved the study protocol (permission nr. HUS/2266/2016). All transplantations were performed adhering to the principles of the Declaration of Helsinki.

Patients were managed as previously described [7]. In brief, the patients received triple-drug primary immunosuppression consisting of a calcineurin inhibitor, mycophenolate mofetil (MMF) and corticosteroids. Dosing of the immunosuppressive drugs was adjusted for renal and bone marrow insufficiency during follow-up as necessary. At our institution, we do not routinely use immunosuppressive induction therapy, and none of the patients in this study received induction. Glomerular filtration rates (GFRs) were measured by clearance of (99)Tc-DTPA preoperatively and during follow-up. Routine controls were performed at regular intervals for the first year after transplantation and included spirometry, bronchoalveolar lavage and transbronchial lung biopsies. Rejection and CLAD were classified and treated according to standard protocol [7].

\section{Genetic analysis}

A saliva sample was given by all patients for genetic testing. Sequence analysis and copy number variation analysis of 77 genes associated with interstitial lung disease was performed at an independent laboratory (Blueprint Genetics, Helsinki, Finland). In supplement 1, a gene list including inheritance mode and variant count of each gene in the Human Gene Mutation Database (HGMD) and ClinVar mutation database is presented. Methods for DNA extraction and sequence analysis are described in supplement 2.

Genetic variants were classified according to the Association for Molecular Pathology/American College of Molecular Genetics and Genomics guidelines [15]. Relying on the genetic standards, a patient was considered to have a molecular diagnosis when a single pathogenic or likely pathogenic variant was seen as heterozygous in a gene associated with dominant disease or when a pathogenic or likely pathogenic pair was seen as homozygous or compound heterozygous in a gene associated with recessive disease. Variants of uncertain significance (VUS) were considered highly suspicious based on: 1) a strong and specific correlation between the gene and patient's phenotype; 2) the variant being novel or extremely rare in the genome aggregation database (gnomAD) or control cohorts; and 3) in silico predictions supporting pathogenicity or the amino acid position in question being highly conserved in mammals and evolutionarily more distant species suggesting that the position does not tolerate variation.

\section{Statistical analysis}

For patient data analysis, the following statistical tests were used: Fischer's exact test for single comparative analysis of categorical variables and contingency analysis, unpaired t-test for continuous variables and log-rank test for analysing event-free survival. 
A molecular diagnosis is established in almost half of lung transplant recipients

15 of the 23 patients (65\%) had a total of 20 rare variants that potentially contributed to the patients' phenotypes (table 1). Of these variants, six were classified as pathogenic, six as likely pathogenic and three as VUSs. 11 of the 23 analysed patients (48\%) received a molecular diagnosis for their IPF (table 1 patient data in bold).

Nine patients with molecular diagnosis had a telomere-related disease: five (45\%) of the diagnostic variants were in the Telomerase Reverse Transcriptase (TERT) gene, two (18\%) in the Poly(A)-Specific Ribonuclease $(P A R N)$ gene and one (9\%) each in the Dyskerin Pseudouridine Synthase 1 (DKC1) and the Telomeric Repeat Binding Factor 1 Interacting Nuclear Factor 2 (TINF2) genes. The patient with a pathogenic variant in TINF2 had a second mosaic variant in the same gene. One patient had a homozygous frameshift variant in the gene for Hermansky-Pudlak Syndrome Protein 1 (HPS1), confirming its diagnosis. One patient was heterozygous for variants in genes for Endothelin 3 (EDN3) and Surfactant Protein A2 (SFTPA2).

The remaining cases were heterozygous variants in the genes Serpin Family A Member 1 (SERPINA1) and Dynein Axonemal Heavy Chain 9 (DNAH9), which are associated with recessive inheritance. None of the patients with SERPINA1 variants had radiographic signs of emphysema before lung transplantation. One patient had a VUS in the gene for Sodium Channel Protein type 4 Subunit Alpha (SCN4A); this patient did not show a phenotype indicating genetic disease.

Enrichment of TERT variants in patients with telomere-related disease and IPF

Two of the observed TERT variants seem to be enriched in Finnish IPF patients. There are 29 individuals heterozygous for the missense variant c.2051A>G, p.(Asp684Gly) among 52188 (0.06\%) individuals in gnomAD. The variant is relatively common especially in Finland as there are 27 heterozygous individuals among 9720 (0.28\%) Finnish individuals. We found two heterozygote cases of this variant, suggesting that it is enriched in lung transplant patients with UIP (OR 32.7, 95\% CI 7,5-124,4, p=0.0021) when compared to the Finnish allele frequency in gnomAD. Previously, the p.(Asp684Gly) variant has been observed as heterozygous in $9.5 \%$ of the patients who underwent genetic testing for interstitial lung disease (unpublished results, Blueprint Genetics) suggesting significant enrichment compared to Finnish controls (OR 35.7, 95\% CI 14.6-82.7, $\mathrm{p}<0.0001$ ).

There are 19 individuals heterozygous for the variant TERT c.2320C $>$ T, p.(Arg774*) in gnomAD. Interestingly, the highest allele frequency is seen in Finland as 16 out of 12,775 (0.13\%) Finnish controls have the variant. Based on the allele frequency in this study $(2 / 46)$, there is enrichment of this variant in Finnish lung transplant recipients with UIP (OR 72.5, 95\% CI 16.1-273.8, p=0.0005). Previously, the variant allele was found in $10.5 \%$ of Finnish patients with interstitial lung disease (unpublished results, Blueprint Genetics). This data supports that this nonsense variant is significantly enriched in these patients (OR 86, 95\% CI 33-224, $\mathrm{p}<0.00001$ ).

\section{Lung transplant recipients with telomere and non-telomere disease have similar outcomes}

We compared patients with telomere disease-causing variants $(n=9)$ and patients with other pathogenic variants or without genetic findings $(n=14)$. Basic clinical characteristics for the two groups are shown in table 2. Patients' ages at the time of diagnosis and transplantation were similar. Telomere-related cases were found in men only, while $64 \%$ of patients were male in the non-telomere disease group $(\mathrm{p}=0.12)$. One patient with TERT c.2320C>T, p.(Arg774*) had a brother with confirmed IPF. Two other patients had a positive family history for IPF but no genetic variant finding in this study $(p=0.99)$. The incidence of comorbidities was similar between the groups.

Outcomes are presented in table 3. Time (mean \pm SD) from diagnosis to lung transplantation was $1100 \pm$ 629 days for patients with telomere-related disease and $1346 \pm 684$ days for the others $(p=0.40)$. Transplant waiting list times and follow-up times were comparable between the groups. During follow-up all nine patients (100\%) with telomeropathy developed renal insufficiency. Of these, eight were mild (GFR 30$60 \mathrm{~mL} \cdot \mathrm{min}^{-1} \cdot 1.73 \mathrm{~m}^{-2}$ ) and only one severe (GFR $<30 \mathrm{~mL} \cdot \mathrm{min}^{-1} \cdot 1.73 \mathrm{~m}^{-2}$ ). In the other group, there were 13 cases $(93 \%)$ of new onset mild renal insufficiency of which one was severe. Leukopenia after the transplant hospitalisation was observed in 8 (57\%) patients in non-telomere-related cases and in 5 (56\%) patients with telomeropathy $(\mathrm{p}=0.99)$. Only one patient $(7 \%)$ without telomere-related disease required permanent withdrawal of MMF. The other cases of leukopenia resolved with dose reduction of MMF or were related to cytomegalovirus re-activation and antiviral therapy. The only malignancies observed in our patients were epidermoid carcinomas of the skin. Its incidence was not statistically different between the 
TABLE 1 Genetic variants in lung transplant recipients with usual interstitial pneumonia

\begin{tabular}{|c|c|c|c|c|c|c|c|}
\hline Patient & Gene (inheritance) & Chromosome position & Variant & Genotype & gnomAD (n) & Age at onset & Classification \\
\hline 1 & TERT (AD/AR) & 5:1279485 & c.2051A>G, p.(Asp684Gly) & HET & 29 & 56 & Pathogenic \\
\hline \multirow[t]{2}{*}{2} & TERT (AD/AR) & $5: 1279485$ & c.2051A>G, p.(Asp684Gly) & HET & 29 & 56 & Pathogenic \\
\hline & SERPINA1 (AR) & 14:94844947 & c.1096G>A, p.(Glu366Lys) & HET & $3054 / 25$ & & Pathogenic \\
\hline \multirow[t]{2}{*}{3} & TERT (AD/AR) & $5: 1272362$ & c. 2320 C $>T$, p. $\left(\operatorname{Arg} 774^{\star}\right)$ & HET & 19 & 57 & Pathogenic \\
\hline & SERPINA1 (AR) & 14:94847262 & c.863A>T, p.(Glu288Val) & HET & $6136 / 132$ & & Pathogenic \\
\hline 4 & TERT (AD/AR) & $5: 1272362$ & c.2320C>T, p. $\left(\operatorname{Arg} 774^{\star}\right)$ & HET & 19 & 55 & Pathogenic \\
\hline 5 & TERT (AD/AR) & 5:1264594 & c.2768C>T, p.(Pro923Leu) & HET & 1 & 63 & Likely pathogenic \\
\hline \multirow[t]{2}{*}{6} & DKC1 (XLR) & X:153999083 & c.965G>T, p.(Arg322Leu) & HEM & 0 & 47 & Likely pathogenic \\
\hline & TERT (AD/AR) & $5: 1279562$ & c.1974G >A, p.(Val658=) & HET & 11 & & VUS \\
\hline 7 & PARN (AD) & 16:14704440-14704726 & c. $\left(388+1 \_389-1\right)$ _(554+1_555-1)del & HET & 0 & 53 & Likely pathogenic \\
\hline 8 & PARN (AD) & 16:14540684-14576744 & c.(1480+1_1481-1)_(1864+1_1865-1)del & HET & 0 & 55 & Likely pathogenic \\
\hline \multirow[t]{2}{*}{9} & TINF2 (AD) & $14: 24709846$ & c. $840 \mathrm{G}>\mathrm{C}, \mathrm{p} .($ Lys 280 Asn $)$ & HET & 0 & 59 & Pathogenic \\
\hline & TINF2 (AD) & $14: 24709920$ & c. $766 C>T$, p. $\left(\operatorname{Arg} 256^{\star}\right)$ & Mosaic & 0 & & Likely pathogenic \\
\hline 10 & HPS1 (AR) & $10: 100186986$ & c.972del, p.(Met325Trpfs*6) & HOM & 26 & 60 & Pathogenic \\
\hline \multirow[t]{2}{*}{11} & EDN3 (AD) & $20: 57876506$ & c.95_106delinsCA, p.(Gly32Alafs*174) & HET & 0 & 43 & Likely pathogenic \\
\hline & SFTPA2 (AD) & 10:81317087 & c.622_624del, p.(Tyr208del) & HET & 0 & & VUS \\
\hline 12 & DNAH9 (AR) & 17:11597289 & c.4719T>G, p.(Tyr1573*) & HET & 57 & 57 & Likely pathogenic \\
\hline 13 & SCN4A (AD) & $17: 62022079$ & c.3866T>C, p.(Leu1289Pro) & HET & 0 & 65 & VUS \\
\hline 14 & SERPINA1 (AR) & 14:94844947 & c.1096G>A, p.(Glu366Lys) & HET & $3054 / 25$ & 51 & Pathogenic \\
\hline 15 & SERPINA1 (AR) & 14:94844947 & c.1096G>A, p.(Glu366Lys) & HET & $3054 / 25$ & 59 & Pathogenic \\
\hline
\end{tabular}

Classifications follow American College of Medical Genetics and Genomic (ACMG) criteria: Pathogenic, Likely pathogenic, VUS. Variants considered molecular diagnoses are in bold. Patients 1-9 had telomere-related disease. AD: autosomal dominant; AD/AR: autosomal dominant and recessive; AR: autosomal recessive; XLR: X-linked recessive; Chr: chromosome; c.: coding DNA sequence; p.: protein sequence; >: substitution; *: termination codon; “=”: synonymous variant; del: deletion; fs: frameshift; delins: deletion/insertion; HET: heterozygote; HOM: homozygote; HEM: hemizygote; gnomAD: genome aggregation database (numbers indicate reported heterozygote/homozygote cases); vUS: variant of uncertain significance. 


\begin{tabular}{|c|c|c|c|}
\hline & Telomere-related & Non-telomere-related" & $\mathrm{p}$-value \\
\hline Age at Dg years (min-max) & $55 \pm 4(47-63)$ & $55 \pm 9(33-68)$ & 0.88 \\
\hline Age at Tx years (min-max) & $59 \pm 5(50-65)$ & $59 \pm 9(39-70)$ & 0.89 \\
\hline Male sex & $9(100)$ & $9(64)$ & 0.12 \\
\hline History of smoking & $7(78)$ & $10(71)$ & 0.99 \\
\hline Family history for IPF & $1(11)$ & $2(14)$ & 0.99 \\
\hline Hypertension & $4(44)$ & $5(36)$ & 0.99 \\
\hline Hyperlipidaemia & $2(22)$ & $1(7)$ & 0.54 \\
\hline Diabetes & $2(22)$ & $0(0)$ & 0.14 \\
\hline CAD & $1(11)$ & $1(7)$ & 0.99 \\
\hline Asthma & $1(11)$ & $0(0)$ & 0.39 \\
\hline Sarcoidosis & $1(11)$ & $0(0)$ & 0.39 \\
\hline Psoriasis & $0(0)$ & $1(7)$ & 0.99 \\
\hline Rheumatoid arthritis & $0(0)$ & $1(7)$ & 0.99 \\
\hline Renal insufficiency $^{+}$ & $0(0)$ & $1(7)$ & 0.99 \\
\hline
\end{tabular}

groups (44\% versus 14\%, $\mathrm{p}=0.16)$. Histopathological acute rejection during the first year after transplantation ( $33 \%$ versus $57 \%$, $\mathrm{p}=0.40$ ) and development of CLAD (33\% versus $43 \%$, $\mathrm{p}=0.99$ ) occurred at a similar rate in both groups. Log-rank test failed to show significant differences for the probability of acute rejection $(\mathrm{p}=0.32)$ or CLAD $(\mathrm{p}=0.42)$ between the groups.

\section{Discussion}

Our results suggest that almost half of the patients with histologically confirmed UIP receiving lung transplants in Finland have a genetic diagnosis possibly contributing to the development of IPF. 39\% of the patients had a molecular diagnosis of telomere disease. A recent report discovered telomere disease-causing variants by using exome sequencing in only $11.8 \%$ of lung transplant recipients [13]. The difference probably highlights globally variable indications for lung transplantation, population differences in genetic contribution and differences between the genetic assays to identify difficult-to-detect genetic variants. We only included patients with confirmed histopathological UIP, and it is possible that genetic variants are over-represented in this group compared to populations with other types of IPF. In Finland, $50 \%$ of lung transplantations are performed on patients with interstitial lung disease [7], while the number in North America ranges from 22 to $32 \%$ [6, 12]. The penetrance of telomere disease-related variants is variable. In this study, we did not measure telomere lengths, which could have provided further evidence that there is a causative relationship between the observed genetic variant and the patients' phenotypes. It is, however, important to point out that patients can express normal pulmonary phenotypes even though

TABLE 3 Outcome of lung transplant recipients with telomere-related and non-telomere-related usual interstitial pneumonia

\begin{tabular}{|c|c|c|c|}
\hline & Telomere-related ${ }^{\#}$ & Non-telomere-related ${ }^{\complement 1}$ & p-value \\
\hline Dg-Tx days (min-max) & $1100 \pm 629(439-2460)$ & $1346 \pm 684(87-2766)$ & 0.40 \\
\hline List time days (min-max) & $82 \pm 99(1-321)$ & $82 \pm 145(0-517)$ & 0.99 \\
\hline Follow-up days (min-max) & $2920 \pm 791(1921-4421)$ & $2473 \pm 1026(1509-4624)$ & 0.28 \\
\hline New onset chronic renal insufficiency & $9(100)$ & $13(93)$ & 0.99 \\
\hline MMF treatment stopped & $0(0)$ & $1(7)$ & 0.99 \\
\hline Skin malignancies & $4(44)$ & $2(14)$ & 0.16 \\
\hline Histopathological rejection & $3(33)$ & $8(57)$ & 0.40 \\
\hline Time to rejection days (min-max) & $59 \pm 15(47-76)$ & $73 \pm 49(31-176)$ & 0.63 \\
\hline CLAD & $3(33)$ & $6(43)$ & 0.99 \\
\hline Time to CLAD days (min-max) & $1475 \pm 329(1102-1724)$ & $1209 \pm 536(469-1832)$ & 0.46 \\
\hline
\end{tabular}


their telomeres are shortened [16, 17]. In addition, patients with IPF often have shortened telomeres even without telomere-related genetic disease [17].

Interestingly, only one of the 11 patients with a molecular diagnosis had a positive family history for IPF, whereas the molecular diagnoses of the remaining 10 patients were novel. Sporadic variants have previously been observed in only one out of $10 \mathrm{IPF}$ patients [16, 17]. It is possible that some familial cases in this study have previously been undiagnosed or that genetic contribution is underestimated or remains undiscovered in general since genetic testing is not utilised routinely in the workup of these patients.

Our study provides statistical evidence that TERT p.(Asp684Gly) and p.(Arg774*) are the most common missense and nonsense variants related to IPF, and that both of the variants are highly enriched in the Finnish population. This may indicate that telomere-related IPF may be more common in Finland than in other countries, but this hypothesis needs further testing by assessing the penetrance of these variants. TERT variants are found in $8-15 \%$ of patients with autosomal dominant familial pulmonary fibrosis [16, 18]. Disease caused by mutations in TERT are inherited in both autosomal dominant and recessive manners but is considered dominant with reduced penetrance in relation to pulmonary fibrosis [17]. In the current study, $26 \%$ of patients had heterozygous TERT variants. The TERT p.(Asp684Gly) variant has previously been observed in one homozygous and two heterozygote individuals out of 135 patients analysed for suspected telomere-related disease [19].

Genetic variants for $P A R N$ are associated with impaired telomere maintenance and are found in $\sim 5 \%$ of cases with familial pulmonary fibrosis [18]. To our knowledge, the observed PARN deletions described here have not been previously published in the relevant medical literature or reported in the disease-related variation databases. There are, however, multiple multi-exon and whole-gene deletions listed in HGMD in patients with telomere shortening-related disease. For PARN c.(1480+1_1481-1)_(1864+1_1865-1)del, in this study there is a partially overlapping de novo deletion (exons 23-24) identified previously in a patient with bone marrow failure and neurological dysfunction [20].

TINF2 encodes a protein that converts uridine to pseudouridine in ribosomal RNA and is part of a complex protecting telomere ends. In familial pulmonary fibrosis, TINF2 variants are observed in $0.6 \%$ of cases. In this study, one patient had two variants for TINF2. The missense variant c.840G $>C$, p.(Lys280Asn) has previously been associated with dyskeratosis congenita [21]. The other c.766C $>\mathrm{T}$, p.(Arg256*) variant was present only in part of the gene reads, suggesting that it was mosaic. Since the DNA sample was extracted from saliva, we could not verify whether the variant was germline mosaic or a somatic mutation. The mosaic variant causes a premature stop codon and loss of normal protein function either through protein truncation or nonsense-mediated mRNA decay from the other allele and might modify the biological mechanism of the other missense variant for this patient as it prevents translation of the other pathogenic missense variant.

HPS1 encodes a transmembrane protein that forms a lysosomal complex together with Hermansky-Pudlak syndrome 4 protein. Variants in HPS1 are associated with autosomal recessive Hermansky-Pudlak syndrome. The syndrome is characterised by albinism, bleeding diathesis, immunodeficiency and pulmonary fibrosis. The variant observed here has previously been found as homozygous in one patient with HPS [22]. In HPS, life-threatening manifestations, such as restrictive lung disease, occur usually starting from the fourth decade of life. There is, however, large variation in disease severity, possibly related to genetic anticipation.

SFTPA2 encodes surfactant protein A2. Surfactant-related genetic variants are thought to increase cellular damage by causing endoplasmic reticulum stress, and genetic variants have been associated with autosomal dominant IPF [23]. The observed variant has not previously been reported, but there was strong association between the mutation and the patient's phenotype. The same patient had a variant for EDN3, encoding Endothelin 3. Variants in this gene have been associated with congenital central hypoventilation syndrome and Waardenburg syndrome. Waardenburg syndrome is characterised by pigmentation abnormalities, hearing loss, musculoskeletal abnormalities and Hirschprung disease [24]. It has not previously been linked to pulmonary fibrosis in the literature but might explain IPF through hypoventilation-related mechanisms. The variant generates a frameshift leading to a premature stop codon in a new reading frame. It is predicted to cause loss of normal protein function either through protein truncation or nonsense-mediated mRNA decay. To our knowledge, the variant has not been published in the relevant medical literature or reported in the disease-related variation databases. Disease caused by EDN3 variants are inherited in both autosomal recessive and dominant manners. The variant was 
considered likely pathogenic considering the established association between the gene and patient's phenotype, rarity in control populations and frameshift mutation type.

DNAH9 encodes a microtubule-associated motor protein. Variants in this gene are associated with primary ciliary dyskinesia and several congenital defects. It is, however, inherited in an autosomal recessive manner and does not cause disease without another disease-causing variant in the same gene [25]. Variants in SCN4A, encoding $\alpha$ subunits of sodium channels, have been associated with disease that causes myotonia or periodic paralysis, and they are inherited in both autosomal recessive and dominant manners. The phenotype is highly variable, and disease onset usually occurs in infancy or early childhood. Muscle and soft tissue symptoms are the most common manifestations [26]. Inspiratory stridor in early life is the only related respiratory disorder that has been reported previously [27].

The variants for SERPINA1 in this study were heterozygous and are not able to cause disease without a second disease-causing variant in the same gene. The p.(Glu366Lys) variant removes a salt bridge to Lys290 and a hydrogen bond to Thr203, causing misfolding of the protein within the endoplasmic reticulum, which results in a lack of secretion from hepatocytes and a reduction of plasma $\alpha$-1-antitrypsin levels to $10-15 \%$ of normal in homozygotes [28] and to $61-77 \%$ in heterozygotes [29, 30]. Heterozygous carrier of p.(Glu366Lys) may have moderately decreased to normal serum $\alpha-1$ antitrypsin levels and could therefore influence pulmonary phenotype.

In this study, we analysed the incidence of monogenetic disease variants and did not include analysis for common gene variants and genes associated with multigenic susceptibility to IPF. Because of the study design, we did not include survival as an end-point. We investigated possible impact of telomere-related variants on disease progression before transplantation and the incidence of acute rejection and CLAD after transplantation. Previously, these variants have been associated with several complications after transplantation but their impact on CLAD development or survival has been controversial [10, 13, 14]. Based on our results, the risk of developing CLAD and time to CLAD onset was not different between patients with telomere-related and non-telomere-related disease. Patient age at disease onset or transplantation, or the time from diagnosis to transplantation did not differ between the groups. This indicates that disease progression was not related to telomeropathy. Leukopenia or other forms of bone marrow dyscrasia is frequent after lung transplantation $[13,14]$. We failed to show any association between bone marrow dysfunction and telomere-related IPF. The overall incidence of post-operative renal dysfunction was more common in this study than in many previous reports, but it was not related to telomere disease. The only malignancies observed in this study were epidermoid skin carcinomas, and they occurred at a similar rate as previously reported [13, 14], with no significant over-representation in telomeropathy patients.

This study has several limitations. It was a retrospective study with small sample size. Patient selection bias is possible because we only included patients that were eligible for lung transplantation and were alive several years after transplantation. These results are not necessarily generalisable to all IPF patients. All patients in this study were included based on the same inclusion criteria, and the two study groups are thereby comparable.

\section{Conclusions}

Sporadic monogenic variants were found in a higher proportion of lung transplant recipients with IPF than previously reported. We identified two variants in the TERT gene that might be enriched in Finnish patients with IPF. This finding could have diagnostic and therapeutic implications in the future but requires further confirmation. In this study, lung transplant recipients with molecular diagnoses for telomere-related disorders seemed to have similar outcomes to patients without confirmed genetic disease susceptibility or non-telomere-related genetic IPF.

Provenance: Submitted article, peer reviewed.

Author contributions: C. Stark recruited the study patients. C. Stark, A. Nykänen, K. L Lemström and P. Raivio gathered patient data. DNA analysis and interpretation were performed by J.W. Koskenvuo, E.H. Seppälä and S. Myllykangas. C. Stark wrote the manuscript. All authors edited and approved the final manuscript.

Conflict of interest: J.W. Koskenvuo, E.H. Seppälä and S. Myllykangas are full-time employees of Blueprint Genetics Ltd. The authors have no others conflicts of interest to declare. 
Support statement: This work was funded by grants from Helsinki University Hospital (grant number Y2019SK017) and Suomen transplantaatiokirurginen yhdistys. Funding information for this article has been deposited with the Crossref Funder Registry.

\section{References}

$1 \quad$ Lederer D, Martinez F. Idiopathic pulmonary fibrosis. N Engl J Med 2018; 378: 1811-1823.

2 Raghu G, Remy-Jardin M, Myers J, et al. Diagnosis of idiopathic pulmonary fibrosis. An official ATS/ERS/JRS/ ALAT clinical practice guideline. Am J Respir Crit Care Med 2018; 198: e44-e68.

3 Ley B, Collard HR, King TE. Clinical course and prediction of survival in idiopathic pulmonary fibrosis. Am J Respir Crit Care Med 2011; 183: 431-440.

4 Kolb M, Bondue B, Pesci A, et al. Acute exacerbations of progressive-fibrosing interstitial lung diseases. Eur Respir Rev 2018; 27: 180071.

5 Raghu G, Rochwerg B, Zhang Y, et al. An official ATS/ERS/JRS/ALAT clinical practice guideline: treatment of idiopathic pulmonary fibrosis: an update of the 2011 clinical practice guideline. Am J Respir Crit Care Med 2015; 192: e3-e19.

6 Christie J, Edwards L, Kucheryavaya A, et al. The registry of the international society for heart and lung transplantation: twenty-seventh official adult lung and heart-lung transplant report. J Heart Lung Trans 2010; 10: P1104-P1118.

7 Nykänen A, Raivio P, Peräkylä L, et al. Incidence and impact of chronic lung allograft dysfunction after lung transplantation - single-center 14-year experience. Scand Cardiovasc J 2020; 54: 192-199.

8 Chambers DC, Cherikh WS, Goldfarb SB, et al. The international thoracic organ transplant registry of the international society for heart and lung transplantation: thirty-fifth adult lung and heart-lung transplant report-2018; focus theme: multiorgan transplantation. J Heart Lung Transplant 2018; 37: 1169-1183.

9 Yanagihara T, Sato S, Upagupta C, et al. What have we learned from basic science studies on idiopathic pulmonary fibrosis? Eur Respir Rev 2019; 28: 190029.

10 Newton C, Molyneaux P, Oldham J. Clinical genetics in interstitial lung disease. Front Med 2018; 5: 116.

11 Arish N, Petukhov D, Wallach-Dayan S. The role of telomerase and telomeres in interstitial lung diseases: from molecules to clinical implications. Int J Mol Sci 2019; 20: 2996.

12 Moua T, Lee A, Ryu J. Comparing effectiveness of prognostic tests in idiopathic pulmonary fibrosis. Expert Rev Respir Med 2019; 13: 993-1004.

13 Swaminathan A, Neely M, Frankel C, et al. Lung transplant outcomes in patients with pulmonary fibrosis with telomere-related gene variants. Chest 2019; 156: 477-485.

14 Tokman S, Singer J, Devine M, et al. Clinical outcomes of lung transplant recipients with telomerase mutations. J Heart Lung Transplant 2015; 34: 1318-1324.

15 Richards S, Aziz N, Bale S, et al. Standards and guidelines for the interpretation of sequence variants: a joint consensus recommendation of the American College of Medical Genetics and Genomics and the Association for Molecular Pathology. Genet Med 2015; 17: 405-424.

16 Armanios $\mathrm{M}$, Chen J, Cogan J, et al. Telomerase mutations in families with idiopathic pulmonary fibrosis. N Engl J Med 2007; 356: 1317-1326.

17 Courtwright A, El-Chemaly S. Telomeres in interstitial lung disease: the short and the long of it. Ann Am Thorac Soc 2019; 16: 175-181.

18 Borie R, Kannengiesser C, de Fontbrune F S, et al. Management of suspected monogenic lung fibrosis in a specialised centre. Eur Respir Rev 2017; 26: 160122.

19 Nordberg A, Rosén A, Raaschou-Jensen K, et al. Novel variants in Nordic patients referred for genetic testing of telomere-related disorders. Eur J Hum Genet 2018; 26: 858-867.

20 Dhanraj S, Gunja SMR, Deveau AP, et al. Bone marrow failure and developmental delay caused by mutations in poly(A)-specific ribonuclease (PARN). J Med Gen 2015; 52: 738-748.

21 Savage SA, Giri N, Baerlocher GM, et al. TINF2, a component of the shelterin telomere protection complex, is mutated in dyskeratosis congenita. Am J Hum Genet 2008; 82: 501-509.

22 Oh J, Ho L, Ala-Mello S, et al. Mutation analysis of patients with Hermansky-Pudlak syndrome: a frameshift hot spot in the HPS gene and apparent locus heterogeneity. Am J Hum Genet 1998; 62: 593-598.

23 Wang $\mathrm{Y}$, Kuan $\mathrm{P}$, Xing $\mathrm{C}$, et al. Genetic defects in surfactant protein A2 are associated with pulmonary fibrosis and lung cancer. Am J Hum Gen 2009; 84: 52-59.

24 Pingault V, Ente D, Dastot-Le Moal F, et al. Review and update of mutations causing Waardenburg syndrome. Hum Mutat 2010; 31: 391-406.

25 Loges N, Antony D, Mayer A, et al. Recessive DNAH9 loss-of-function mutations cause laterality defects and subtle respiratory ciliary-beating defects. Am J Hum Gen 2018; 103: 995-1008.

26 Dupré N, Chrestian N, Bouchard JP, et al. Clinical, electrophysiologic, and genetic study of non-dystrophic myotonia in French-Canadians. Neuromuscul Disord 2009; 19: 330-334.

27 Matthews E, Manzur A, Sud Y, et al. Stridor as a neonatal presentation of skeletal muscle sodium channelopathy. Arch Neurol 2011; 68: 127-129. 
28 Hughes VA, Meklemburg R, Bottomley SP, et al. The Z mutation alters the global structural dynamics of a1-antitrypsin. PLOS ONE 2014; 9: e102617.

29 Calapoglu M, Deger O, Balaban F, et al. Analysis of the alpha1antitrypsin deficient alleles M3S, MZ, and ZZ by biochemical and molecular methods: a family study. Biochem Genet 2009; 47: 33-41.

30 Bornhorst J, Greene D, Ashwood E, et al. $\alpha$-1-antitrypsin phenotypes and associated serum protein concentrations in a large clinical population. Chest 2013; 143: 1000-1008. 